\title{
Approach to Wide-Frequency Battery Impedance Measurements in Commercial Applications
}

\author{
Arne Sandschulte, Roberto Ferrero \\ Dept. of Electrical Engineering \& Electronics \\ University of Liverpool \\ Liverpool, UK \\ Arne.Sandschulte@liverpool.ac.uk \\ Roberto.Ferrero@liverpool.ac.uk
}

\author{
Laurence Hardwick \\ Stephenson Institute for Renewable Energy \\ University of Liverpool \\ Liverpool, UK \\ Laurence.Hardwick@liverpool.ac.uk
}

\author{
Edoardo Patelli \\ Institute for Risk \& Uncertainty \\ University of Liverpool \\ Liverpool, UK \\ Edoardo.Patelli@liverpool.ac.uk
}

\begin{abstract}
Electrochemical Impedance Spectroscopy (EIS) is a well-established diagnostic technique for the characterization and monitoring of electrochemical devices, such as batteries. As different electrochemical processes have very different dynamics, a complete characterization of batteries requires measuring their impedance over a very wide frequency range, from less than $1 \mathrm{mHz}$ to more than $1 \mathrm{kHz}$. However, significant measurement challenges affect the extreme frequencies and may limit the practically achievable frequency range, particularly in case of insitu EIS. This paper analyzes the main measurement challenges that appear at low and high frequencies, respectively, and proposes possible strategies to address them, suitable to be used in commercial applications. In more detail, a compensation for the effect of the state-of-charge variation is proposed to improve the accuracy of EIS at low frequencies, while a strategy to measure high-frequency perturbations with a lower sampling frequency is presented to achieve high-frequency EIS with affordable instrumentation. Experimental results obtained on a $\mathrm{LiFePO4}$ cell are reported, used to validate the proposed methods and discuss their limitations.
\end{abstract}

Index Terms-Batteries, Electrochemical devices, Electrochemical impedance spectroscopy, Measurement, Condition monitoring, State estimation

\section{INTRODUCTION}

The state and dynamic behavior of batteries, as well as other electrochemical power sources, is characterized by a complex interplay of several physical and chemical processes, which evolve on very different time scales, from microseconds to years [1]. An effective method to analyze those processes and distinguish between their different contributions to the electrical output of the battery is the Electrochemical Impedance Spectroscopy (EIS): An AC perturbation is created in the battery current (or voltage), at a certain frequency, and the voltage (or current) response to that perturbation is measured, from which the impedance at that frequency is determined. Measuring the impedance at different frequencies allows characterizing different processes, from the fast electric response (ohmic phenomena) to the slower charge transfer and mass transport processes [1]. A generic illustration of a typical battery impedance spectrum is reported in Fig. 1.

This work was partially supported by funding from the Department for Transport, UK (grant T-TRIG-OC059) and from the Royal Society, UK (grant $\mathrm{RSG} \backslash \mathrm{R} 1 \backslash 180328)$.

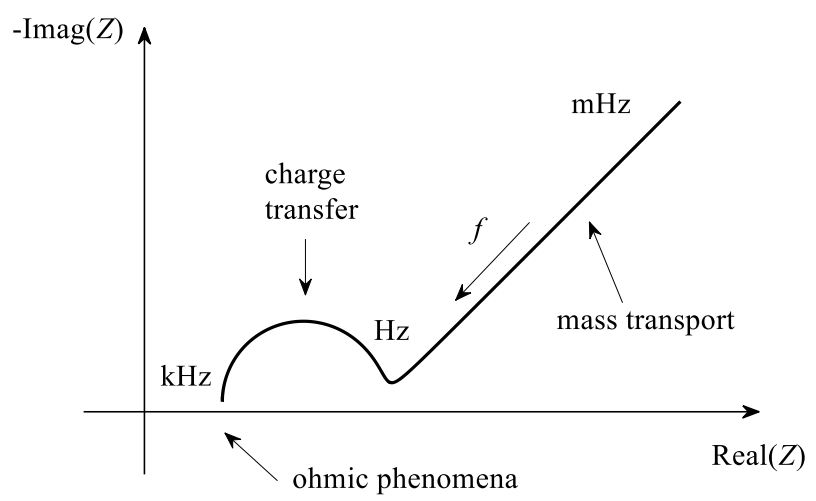

Fig. 1. Illustration of a generic battery impedance spectrum.

EIS has been widely investigated and used in the last couple of decades, and it has informed the development of battery models, particularly in terms of equivalent electrical circuits [2]-[5] ***UPDATE REFS***. Battery impedance spectra reported in the literature cover frequencies down to a few millihertz (in some cases even down to a few microhertz), and up to a few kilohertz or tens of kilohertz ***ADD REFS***. Such extreme frequency ranges give rise to different types of measurement challenges. At low frequencies, transients in the battery state represent the main issue. By definition, the concept of impedance relies on the assumption of steady-state AC conditions; this means that, ideally, the battery should not undergo any change in its internal state, including in particular its state of charge, during the whole time required to measure the impedance, which can be up to several minutes or even hours at the low frequencies mentioned above. This may be feasible under accurately controlled laboratory conditions, and with no DC current generated/absorbed by the battery, but it becomes unfeasible in case of in-situ measurements. On the other hand, high-frequency impedance measurements normally require instruments with a high sampling frequency to acquire the high-frequency perturbations in the battery voltage and current. While those instruments are commonly available in a laboratory environment, they may not be affordable in commercial applications due to cost and complexity constraints. 
To date, most of the EIS measurements reported in the literature have been carried out in the laboratory, mainly with the aim to improve battery models, characterize material properties, or investigate degradation phenomena. The practical relevance of the challenges arising from in-situ EIS has therefore been limited. However, recent works have proposed solutions to effectively achieve EIS in commercial applications, by using power converters to create the required $\mathrm{AC}$ perturbations, in place of the dedicated instrumentation commonly used in the laboratory [6]-[14] ***UPDATE REFS***. In most applications, batteries are connected to a switch-mode DC$\mathrm{DC}$ converter; the AC perturbation in the battery current (or voltage) can therefore be conveniently created by modulating the duty cycle of the converter switching function. As most DC-DC converters nowadays reach switching frequencies in the range of tens or hundreds of kilohertz (particularly at low power), $\mathrm{AC}$ perturbations can cover the whole frequency range required for a complete EIS characterization, or most of it, on the proviso that the converter design allows small highfrequency components on the battery side (i.e. it does not filter them out completely). Therefore, the method itself does not introduce any significant limitation to the EIS frequency range, but the challenges mentioned above can practically limit it. Indeed, feasibility studies of converter-based EIS reported in the literature to date have focused on a narrow frequency range and on small-scale systems (one or a few cells) ***ADD REFS***.

This paper analyzes the main challenges that are likely to hinder low-frequency and high-frequency impedance measurements, respectively, and proposes strategies to effectively address some of those challenges in commercial applications and to assess the validity of EIS results. The assumptions underlying impedance measurements are firstly reviewed in Sec. II, together with some considerations on their practical impact on EIS. The challenges and proposed strategies for low-frequency and high-frequency EIS are then presented in Secs. IV and V, respectively, after describing the experimental setup used for the experimental validation in Sec. III.

\section{Battery Model And ImPedance Measurement}

Fig. 2a shows a generic equivalent circuit model (ECM) describing an electrochemical cell. It consists of a source of electromotive force (EMF) in series with an internal impedance. The value of the EMF can be measured under open circuit conditions as the open circuit voltage, $V_{O C V}$, whereas the impedance models the internal voltage drop appearing when current is drawn from the cell, caused by the various phenomena mentioned above.

Both EMF and internal impedance are functions of the battery state of charge (SoC); additionally, the impedance is a function of the current drawn from the cell, as the I-V characteristic of the battery is nonlinear. These two aspects pose significant challenges for EIS. It is therefore crucial to define the conditions that must be satisfied for a valid impedance measurement.

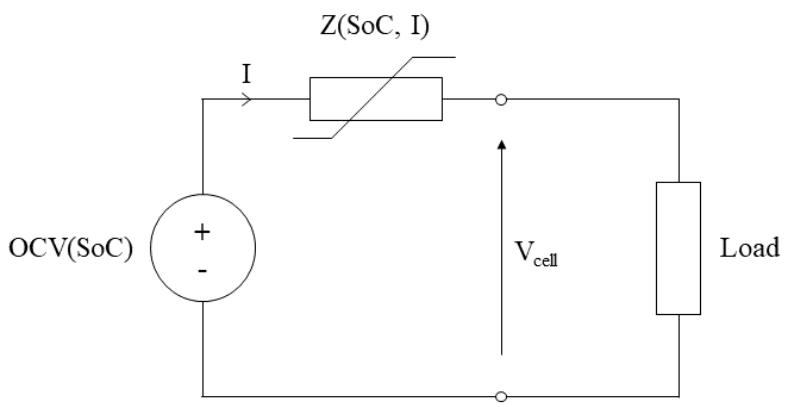

(a)

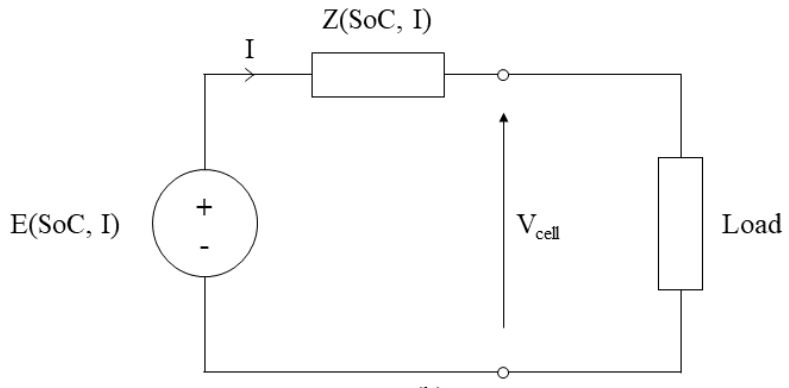

(b)

Fig. 2. Illustration of a generic nonlinear equivalent circuit of a battery connected to a load (a) and the corresponding linearized circuit (b).

Impedance is generally defined for a linear circuit element, in AC steady-state conditions, as the complex ratio between voltage and current phasors. The first condition for a valid measurement is therefore that the system is in steady state, which means that any SoC variations and any other transients must be absent or negligible during the whole measurement period. Moreover, since the impedance is defined as a ratio between phasors, it is only valid for linear time-invariant systems, whose response to a sinusoidal perturbation is sinusoidal, at the same frequency. However, a nonlinear system can still be modeled in terms of impedance if it is subjected to small sinusoidal perturbations within a restricted part of its I-V characteristic that can be approximated by a straight line.

To correctly interpret impedance measurements for an electrochemical cell, it is therefore necessary to linearize the circuit of Fig. 2a around a specified operating point, characterized by a well-defined SoC and a DC current (or voltage), under the assumption that the AC perturbation will cause only a small deviation from the specified point. The resulting circuit (shown in Fig. 2b) will have an equivalent EMF and an equivalent series impedance, which both depend on the operating point.

Assuming, without loss of generality, that the impedance is measured by controlling the current and measuring the voltage response (galvanostatic mode), the generic current drawn from the battery, containing both DC and AC (sinusoidal) components, can be written as:

$$
i(t)=I_{D C}+I_{A C} \sin (2 \pi f t)
$$

where $I_{D C}$ and $I_{A C}$ are the amplitudes of the DC and $\mathrm{AC}$ components, respectively, $f$ is the frequency of the $\mathrm{AC}$ 
component, and its phase is arbitrarily chosen to be zero.

The validity of the assumptions required for a meaningful impedance measurement depends on the values of $I_{D C}, I_{A C}$ and $f$. In more detail, $I_{A C}$ must be small enough to satisfy the linearization assumptions, while the steady-state condition requires the SoC variations caused by the current to be small too.

The shortest possible measurement time is equal to the $\mathrm{AC}$ period, i.e. $1 / f$. During this time, the DC current will cause a linear SoC variation, whose amplitude is:

$$
\Delta \mathrm{SoC}_{D C}(\%)=\frac{I_{D C}}{f Q_{\text {total }}} \times 100
$$

where $Q_{\text {total }}$ is the rated cell capacity. On the other hand, the AC current will cause a sinusoidal oscillation in the SoC, whose peak-to-peak amplitude is:

$$
\Delta \mathrm{SoC}_{A C}(\%)=\frac{I_{A C}}{\pi f Q_{\text {total }}} \times 100
$$

In both cases, the $\mathrm{SoC}$ variation is proportional to the current amplitude and inversely proportional to the frequency of the $\mathrm{AC}$ component. It is therefore evident that keeping the SoC variation small is more challenging at lower frequencies; moreover, also the nonlinear behavior of the battery is enhanced at lower frequencies. Therefore, satisfying both the steady-state condition and linearization requirements is particularly critical for low-frequency EIS measurements.

The DC current component is not needed for impedance measurements, and indeed laboratory-based EIS is often performed with zero DC current, thus completely eliminating the SoC variation caused by it. However, this solution may be difficult (if not impossible) to achieve in in-situ EIS, because batteries are normally operated (charged or discharged) with a significant DC current component, and the power converters connected to them may not allow producing a sinusoidal perturbation with bidirectional current flow. Therefore, lowfrequency in-situ EIS requires strategies to effectively deal with large DC components and the associated SoC variation, which will be discussed in Sec. IV.

The AC current component, on the other hand, can and should be as small as possible, not only to keep the SoC variation limited, but also to minimize ohmic losses and to reduce the effect of an oscillating power supplied to the load connected to the battery. The only limitation to lowering the amplitude of the AC component is the decrease of the signalto-noise ratio (SNR), which may affect the measurement accuracy, so a compromise is required. Again, this may be more challenging to achieve in in-situ EIS, because the environment is likely to be much more noisy than a laboratory.

According to the equivalent circuit in Fig. 2b, the impedance at frequency $f$ is estimated by measuring the terminal voltage response to the current perturbation in (1) and extracting the $\mathrm{AC}$ component from it by removing the DC component, which includes the EMF and the response to $I_{D C}$. Any SoC variation during the measurement time would have two undesired effects: It would create a transient in the EMF and

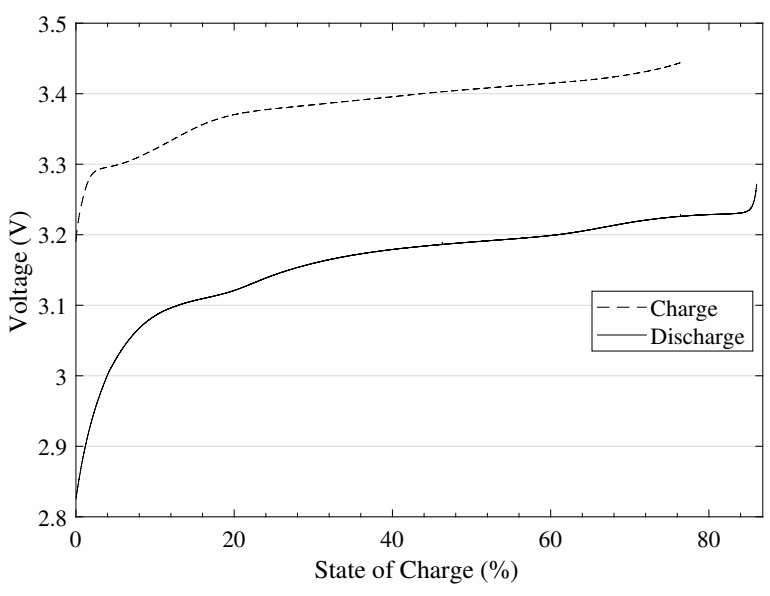

Fig. 3. Charge and discharge curves of a K2 LFP cell measured with a DC current of $0.5 \mathrm{~A}$, corresponding to a C-rate of approximately 0.16 .

potentially change the impedance itself, as both the EMF and the impedance are functions of the SoC. Although both effects would violate the requirements for impedance measurements discussed above, a transient in the EMF alone can be effectively managed by suitable signal processing, whereas a significant change in the impedance would completely jeopardize the validity of the impedance measurement. It is therefore important to distinguish between these two effects. A deeper discussion is presented in Sec. IV, with the support of experimental results.

\section{EXPERIMENTAL SETUP}

The cell used in the experimental investigation reported in this paper is the K226650E02 manufactured by K2 Energy. This is a cylindrical 26650 Lithium Iron Phosphate (LFP) cell with a nominal capacity of $3.2 \mathrm{Ah}$ at $\mathrm{C} / 5$.

Fig. 3 shows the cell's charge and discharge curves measured with a DC current of $0.5 \mathrm{~A}$, equivalent to a C-rate of approximately 0.16. Apart from the extreme parts of the curves, which are highly nonlinear, the two curves can be well approximated by a linear relationship between the $\mathrm{SoC}$ and the terminal voltage. Such a linear approximation can be used to estimate the change of the battery EMF caused by a change in the SoC, i.e. $\frac{\Delta V_{O C V}}{\triangle S o C}$, which results to be $1.7 \mathrm{mV} / \mathrm{SoC}(\%)$ for the discharge curve and $1.1 \mathrm{mV} / \mathrm{SoC}(\%)$ for the charge curve.

To perform EIS measurements, the cell is connected to either a programmable DC electronic load (Elektro Automatik EL 9200-70 B) or a fully controllable DC-DC boost converter connected to a resistive load. The electronic load is typically used in laboratory EIS, whereas the DC-DC converter is suitable for in-situ EIS in commercial applications. Both the electronic load and the DC-DC converter are controlled by an embedded controller (National Instruments CompactRIO 9035) with analog and digital input/output modules. The same system is used to acquire the battery voltage and current signals, with sampling frequencies up to $100 \mathrm{kSa} / \mathrm{s}$. The current measurement is obtained from a Hall-effect closed- 


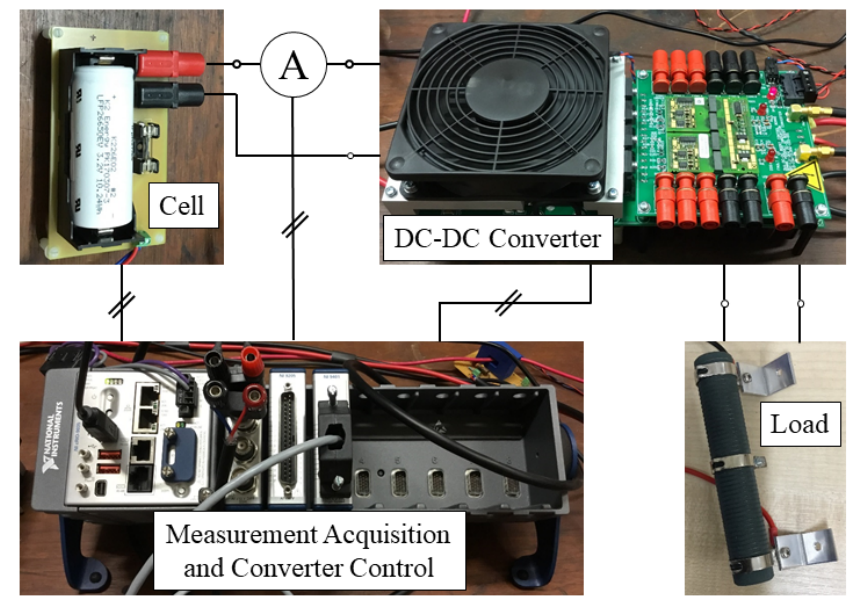

Fig. 4. Block diagram of experimental setup.

loop current transducer (LEM LA 25-P). A block diagram of the whole experimental setup (with the DC-DC converter) is shown in Fig. 4.

\section{Low-Frequency Impedance Measurements}

Low-frequency impedance measurements require a long measurement time, during which the state of the battery should remain as steady as possible, as explained in Sec. II. The use of power converters allows performing EIS either during battery discharge or charge. However, in practice, the charge process presents more favorable conditions because the discharge is often characterized by large transients, due to load variations, whereas the battery current is kept constant during most of the charging time and it usually has a lower amplitude than the rated discharge current. Low-frequency impedance measurements are therefore more likely to provide accurate results if performed while charging the battery.

In this section, the measurement of the battery impedance at $1 \mathrm{mHz}$ during the battery charge is reported as an example to discuss the effects of $\mathrm{SoC}$ variations on low-frequency measurements and to propose possible methods to assess the validity of the results and to improve their accuracy, when possible. The electronic load is used to control the current supplied by an external DC voltage source to the battery, in order to have a sinusoidal component at $1 \mathrm{mHz}$, with $50 \mathrm{~mA}$ amplitude, superimposed onto a DC current of $0.5 \mathrm{~A}$, according to (1).

Fig. 5 shows the cell's voltage response in the time domain, acquired over one period of the AC component (1000 s), and the corresponding voltage spectrum. A linear, time-invariant system, in steady-state condition, perturbed with a sinusoidal current, would have shown a sinusoidal response at the same frequency, from which the impedance at that frequency could be calculated. The spectrum in Fig. 5, however, clearly shows other significant frequency components, indicating that the conditions above are not satisfied. It is worth noting that the signal acquisition is synchronized with the waveform generation, therefore there is no spectral leakage caused by
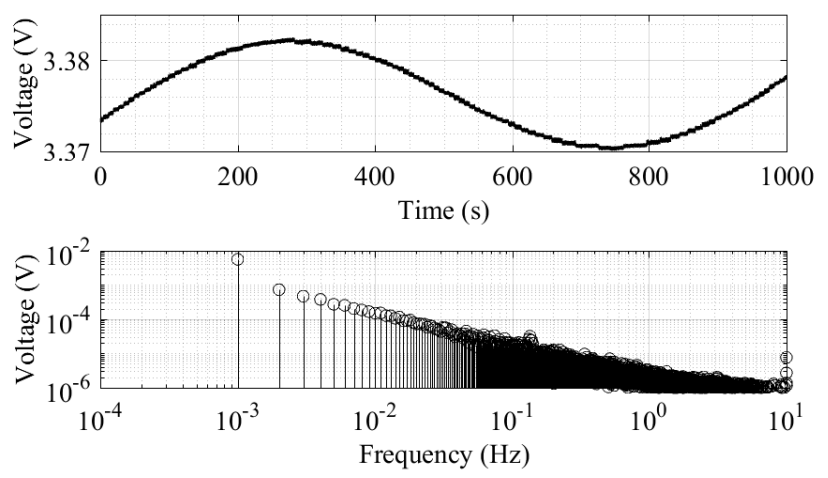

Fig. 5. Voltage waveform in the time domain, and corresponding spectrum in the frequency domain.

a lack of synchronism. Reasons for the observed frequency components can be a nonlinear response of the battery, a transient voltage superimposed to the voltage drop on the equivalent impedance, or a variation of the impedance during the measurement time. All of them are possible, according to what explained in Sec. II, and should be carefully analyzed, as they affect the validity of the impedance estimation in different ways.

From (2), it can be determined that the DC component of the current causes a change in $\mathrm{SoC}$ of approximately $4.3 \%$ during the measurement time. Using the $\frac{\Delta V_{O C V}}{\triangle S o C}$ figure obtained from the charge curve in Sec. III, this is found to correspond to a change in $V_{O C V}$ of approximately $5 \mathrm{mV}$. This is a considerable change which results in a significant rising drift in the measured voltage, as can be seen in the time-domain response of Fig. 5. Such a drift is almost linear (except in the extreme parts of the charge curve), therefore it can be removed from the measured voltage by fitting the curve in the time-domain with a ramp superimposed to a sine wave, using a least-squares method. The resulting signal, shown in Fig. 6, is much closer to a pure sinusoidal waveform. The amplitudes of the second and third harmonics of the $1 \mathrm{mHz}$ have been reduced by approximately $31 \mathrm{~dB}$ and $28 \mathrm{~dB}$, respectively, and all harmonics are now below $0.3 \%$ of the fundamental component. Assuming the resultant spectral line at $1 \mathrm{mHz}$ in Fig. 6 as the best estimate of the actual value, the uncompensated linear drift would have led to an error of $21 \%$ on its amplitude and 0.9 degrees on its phase.

It is important to note that removing the drift caused by the SoC variation leads to valid results only under the assumption that the SoC variation affected only the EMF in the equivalent circuit of Fig. 2b, and not the impedance itself. Any significant impedance change during the measurement time would create a distortion in the voltage response and would not allow using the Fourier Transform to estimate the impedance; in fact, there would be no defined impedance value. It is known that the battery impedance changes as a function of the SoC ***ADD REFS***, but the change may be negligible if the SoC variation is limited. This assumption can be justified a 

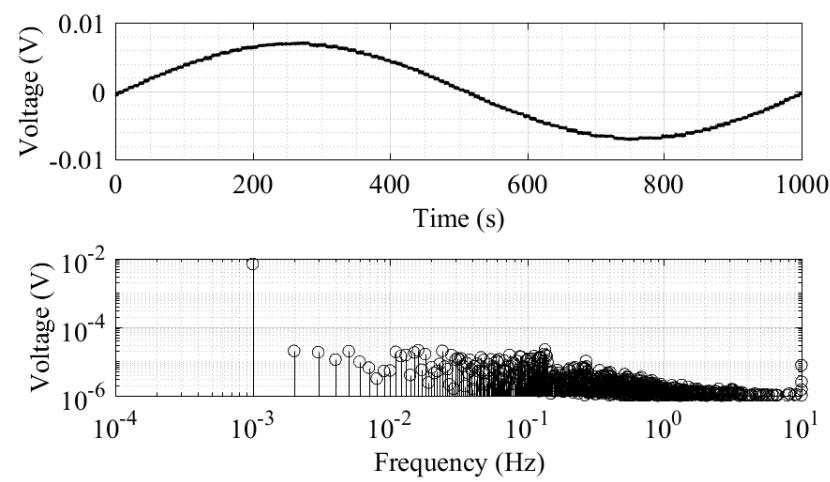

Fig. 6. Voltage waveform in the time domain after removing the linear drift, and corresponding spectrum in the frequency domain.

posteriori by observing the lack of distortion in the voltage signal in Fig. 6. Similarly, the lack of distortion confirms also the validity of the approximation of linear response.

There is, however, another important aspect that must be considered to confirm the validity of the impedance estimate based on the voltage waveform reported in Fig. 6, i.e. the variation of the SoC caused by the AC current component. From (3), it can be seen that a peak-to-peak SoC variation of approximately $0.14 \%$ is expected for a $1 \mathrm{mHz}$ current of $50 \mathrm{~mA}$ amplitude. This is much smaller than the $\mathrm{SoC}$ variation caused by the DC current, but it causes an oscillation in the voltage at the same frequency as the AC perturbation, and it is therefore impossible to distinguish from the voltage drop on the impedance. Such a voltage oscillation may lead to very large errors in the impedance estimate (both magnitude and phase), unless its amplitude is negligible compared to the voltage drop on the impedance, or the oscillation is removed from the signal. It is therefore crucial to estimate the amplitude of the oscillation. A rough estimate can be obtained again from the $\frac{\Delta V_{O C V}}{\Delta S o C}$ figure used above, and it results to be $160 \mu \mathrm{V}$, i.e. approximately $1.1 \%$ of the amplitude of the measured voltage waveform. This amplitude is negligible in this case, but a more significant oscillation could have been removed from the measured waveform, based on the estimate of its amplitude and knowing that it has a 90 degrees phase difference from the current (as it is in phase with the charge oscillation that is the integral of the current).

\section{High-Frequency ImPedanCE Measurements}

Battery packs used in most commercial applications are composed of several tens or hundreds of cells, with a combination of series and parallel connections. Accurately monitoring each cell, or at least small groups of cells, requires to acquire a large number of voltage and current signals; this is only affordable if multiplexed analog-to-digital converters (ADCs) are used, with several input channels connected to a single ADC. Moreover, the small amplitude of the cell voltage variation requires a good resolution of the ADC to accurately measure the small AC voltage response during EIS. These two requirements, together, limit the maximum sampling frequency that can be practically achieved in commercial applications, and may therefore hinder high-frequency impedance measurements.

According to the sampling theorem, a signal with frequency components up to $f_{\max }$ requires a sampling frequency higher than $2 f_{\max }$ to preserve the information contained in the original signal; unless this condition is satisfied, aliasing errors occur and the signal can no longer be correctly reconstructed from its samples. However, this requirement can be circumvented if the signal is periodic and stationary, and its frequency content is perfectly known. If the sampling frequency and the observation time are properly chosen, the aliasing errors will change the frequencies of the sinusoidal components of the signal (in a predictable way), but will leave their amplitudes unchanged. As long as different frequency components do not overlap with each other, the aliased spectra of the measured voltage and current waveforms can still be used to correctly estimate the impedance at the various frequency components present in the original signal. It is worth noting that the aliasing will change the phases of both voltage and current signals, but their difference (i.e. the impedance angle) will remain the same.

This technique can be effectively used to achieve highfrequency EIS with a limited sampling frequency, on the proviso that the conditions above are strictly satisfied. In particular, in order to know the spectral content of the signal exactly, the acquisition must be synchronized with the AC waveform generation, and the voltage and current signals must be cleaned from all transients.

The proposed approach is illustrated here with the experimental setup described in Sec. III. The battery is connected to the DC-DC converter and the duty cycle of the converter is modulated with a small multi-sine oscillation, with frequency components at $100.5 \mathrm{~Hz}$ and $1000.5 \mathrm{~Hz}$. This modulation creates the same frequency components in the battery current and voltage, as seen in the spectra reported in Fig. 7, obtained by sampling both signals at a frequency of $100 \mathrm{kHz}$ (therefore, with no aliasing), over a time of $10 \mathrm{~s}$.

If the same signals are down-sampled at a much lower frequency of $8 \mathrm{~Hz}$, both frequency components will be affected by aliasing errors, and they will appear at completely different frequencies in the current and voltage spectra, shown in Fig. 8. Nevertheless, the impedance values at the two frequencies, estimated from the aliased spectra, are similar to the (more accurate) values obtained from the original spectra. A comparison is reported in Table I.

Some important points should be noted: The accuracy of the impedance estimation has worsened with the down-sampling because also the noise was affected by aliasing and the SNR has effectively decreased; however, the effect of noise can be reduced by averaging results over time. Moreover, interference from nearby power equipment (e.g. operated at $50 \mathrm{~Hz}$ ) may be present in the signals, as shown in the current spectra in Figs. 7 and 8 , so a suitable sampling frequency should be chosen to avoid any overlapping between the $50 \mathrm{~Hz}$ (and its harmonics) and the frequencies of interest in the aliased spectra. 

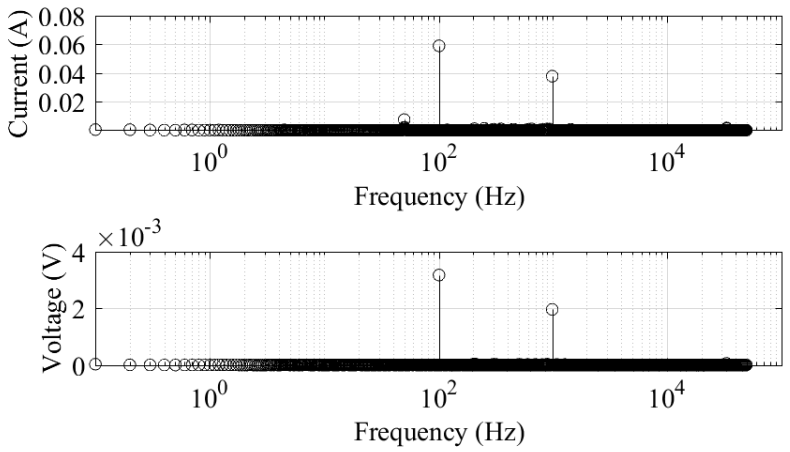

Fig. 7. Frequency spectra of the current and voltage signals sampled at $100 \mathrm{kHz}$, with no aliasing.
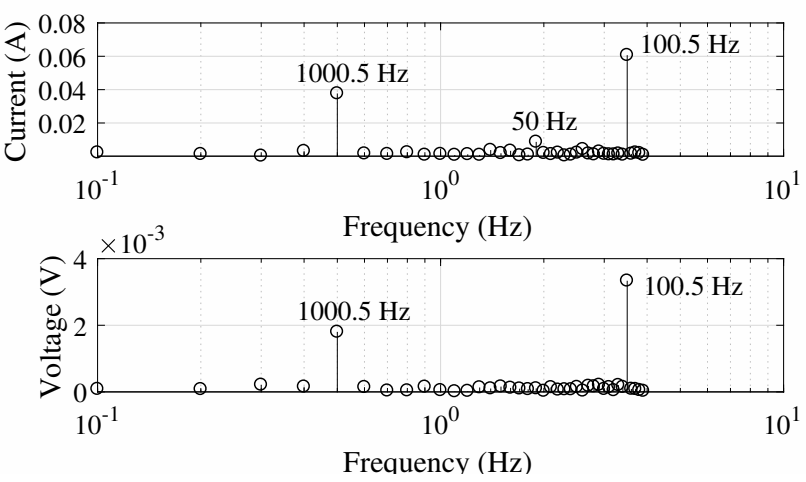

Fig. 8. Frequency spectra of the current and voltage signals sampled at $8 \mathrm{~Hz}$, with aliasing.

\section{CONCLUSIONS}

This paper discussed some important measurement challenges that are likely to affect battery impedance measurements at very low and very high frequencies, particularly when those measurements are performed in-situ, during the battery operation (charge or discharge). At low frequencies, the main challenge arises from the validity of the impedance concept, which requires steady-state conditions and a linear time-invariant response of the battery. Strategies to better satisfy these conditions and assess their validity were proposed, and limitations were discussed. On the other hand, highfrequency measurements may be hindered by the limited sam-

TABLE I

Comparison Between Impedance Measurements Obtained with HigH VS LOW SAMPLING FREQUENCIES.

\begin{tabular}{|l|l|l|}
\cline { 2 - 3 } \multicolumn{1}{c|}{} & $f_{s}=100 \mathrm{kHz}$ & \multicolumn{1}{c|}{$f_{s}=8 \mathrm{~Hz}$} \\
\hline \multicolumn{3}{|c|}{ Impedance at $100.5 \mathrm{~Hz}$} \\
\hline$|Z|$ & $53.7 \mathrm{~m} \Omega$ & $54.9 \mathrm{~m} \Omega$ \\
$\angle Z$ & $-4.7^{\circ}$ & $5.7^{\circ}$ \\
\hline \multicolumn{3}{|c|}{ Impedance at $1000.5 \mathrm{~Hz}$} \\
\hline$|Z|$ & $51.9 \mathrm{~m} \Omega$ & $47.8 \mathrm{~m} \Omega$ \\
$\angle Z$ & $14.6^{\circ}$ & $8.6^{\circ}$ \\
\hline
\end{tabular}

pling frequency available in commercial applications, for the acquisition of voltage and current signals. Possible methods to effectively use a lower sampling frequency to achieve the highfrequency impedance measurements were presented, together with their limitations. All proposed approaches were validated through an experimental investigation on a $\mathrm{LiFePO} 4$ cell.

\section{REFERENCES}

[1] A. Jossen, "Fundamentals of battery dynamics", Journal of Power Sources, vol. 154, pp. 530-538, 2006.

[2] W. Waag, S. Käbitz and D. U. Sauer, "Experimental investigation of the lithium-ion battery impedance characteristic at various conditions and aging states and its influence on the application", Applied Energy, vol. 102, pp. 885-897, 2013.

[3] D. Andre, M. Meiler, K. Steiner, Ch. Wimmer, T. Soczka-Guth and D. U. Sauer, "Characterization of high-power lithium-ion batteries by electrochemical impedance spectroscopy. I. Experimental investigation", Journal of Power Sources, vol. 196, pp. 5334-5341, 2011.

[4] U. Tröltzsch, O. Kanoun and H.-R. Tränkler, "Characterizing aging effects of lithium ion batteries by impedance spectroscopy", Electrochimica Acta, vol. 51, pp. 1664-1672, 2006.

[5] F. Huet, "A review of impedance measurements for determination of the state-of-charge or state-of-health of secondary batteries", Journal of Power Sources, vol. 70, pp. 59-69, 1998.

[6] M. Shirazi, J. Morroni, A. Dolgov, R. Zane and D. Maksimovic, "Integration of frequency response measurement capabilities in digital controllers for DC-DC converters", IEEE Trans. on Power Electronics, vol. 23 , no. 5 , pp. 2524-2535, 2008.

[7] D. Depernet, O. Ba and A. Berthon, "Online impedance spectroscopy of lead acid batteries for storage management of a standalone power plant", Journal of Power Sources, vol. 219, pp. 65-74, 2012.

[8] W. Huang and J. A. Abu Qahouq, "An online battery impedance measurement method using DC-DC power converter control", IEEE Trans. on Industrial Electronics, vol. 61, no. 11, pp. 5987-5995, 2014.

[9] A. Densmore and M. Hanif, "Determining battery SoC using electrochemical impedance spectroscopy and the extreme learning machine", IEEE 2nd Int. Future Energy Electronics Conference, Taipei, Taiwan, 1-4 Nov. 2015, pp. 1-7.

[10] M. A. Varnosfaderani and D. Strickland, "Online impedance spectroscopy estimation of a battery", 18th European Conference on Power Electronics and Applications, Karlsruhe, Germany, 5-9 Sep. 2016, pp. $1-10$.

[11] E. Din, C. Schaef, K. Moffat and J. T. Stauth, "A scalable active battery management system with embedded real-time electrochemical impedance spectroscopy", IEEE Trans. on Power Electronics, vol. 32, no. 7, pp. 5688-5698, 2017.

[12] R. Ferrero, C. Wu, M. De Angelis, H. George-Williams, E. Patelli, A. Carboni, S. Toscani and P. A. Pegoraro, "Low-cost battery monitoring by converter-based electrochemical impedance spectroscopy", IEEE Int. Workshop on Applied Measurements for Power Systems (AMPS), Liverpool, UK, 20-22 Sep. 2017, pp. 1-6.

[13] G. Dotelli, R. Ferrero, P. Gallo Stampino, S. Latorrata and S. Toscani, "PEM fuel cell drying and flooding diagnosis with signals injected by a power converter", IEEE Trans. on Instrumentation and Measurement, vol. 64 , no. 8, pp. 2064-2071, 2015.

[14] G. Dotelli, R. Ferrero, P. Gallo Stampino, S. Latorrata and S. Toscani, "Low-cost PEM fuel cell diagnosis based on power converter ripple with hysteresis control", IEEE Trans. on Instrumentation and Measurement, vol. 64, no. 11, pp. 2900-2907, 2015. 